\title{
QR Code with Colored Image
}

\author{
Kanchan S. Jahagirdar \\ Late G N Sapkal College of Engineering, \\ Nashik
}

\author{
S.B. Borse \\ Late G N Sapkal College of Engineering, \\ Nashik
}

\begin{abstract}
It is a direct method to insert vital information using QR codes into color images with minimal probability of error detection. With standard decoding applications these insertions of vital information using QR code into color codes are suitable. It takes benefits of the support of QR readers against interruption of image luminance moreover, the vital information in $\mathrm{QR}$ code bits are transformed into the luminance values of the image. Multiresolution halftoning masks algorithm uses for the selection of modified pixels and nonlinear programming techniques with which pixel of the images are transformed into luminance level, to reduce the visual distortion of the $\mathrm{QR}$ image. Here a $\mathrm{QR}$ code with colored image is proposed with new multiresolution technique to minimize processing time.
\end{abstract}

\section{General Terms}

Quick response, Multiresolution halftoning

\section{Keywords}

Quick response, Multiresolution halftoning

\section{INTRODUCTION}

A QR code is two dimensional barcode which encodes much information such as, number, binary codes; letters etc [1].QR code can store more information than one dimensional barcode. In horizontal and vertical direction of the QR code it stores the vital information. Maximum storage capacity of the QR code is 7089 numeric, 4296 binary character, and 2953 alphanumeric character [2].

There are 40 version of the $Q R$ code determines its size and it goes from $21 \times 21$ modules for version 1 up to $177 \times 177$.During calculations of errors the QR codes use Reed Solomon code and there are 4 types of error correction L, M, Q and $\mathrm{H}$ that allow correcting up to $7 \%, 15 \%, 20 \%$ and $30 \%$ of code words in error respectively. Different types of QR codes defined in the standard are identified by their error correction level and version. QR codes have square shapes and limited color tolerance which in an important issue in the QR code [4]. Because of its shape and limitation of colors the QR code produces more interest for algorithm which being able to insert vital information present in the $\mathrm{QR}$ codes into the colored images. QR code has perfect pattern and structure. $\mathrm{QR}$ code structure is shown in figure1.

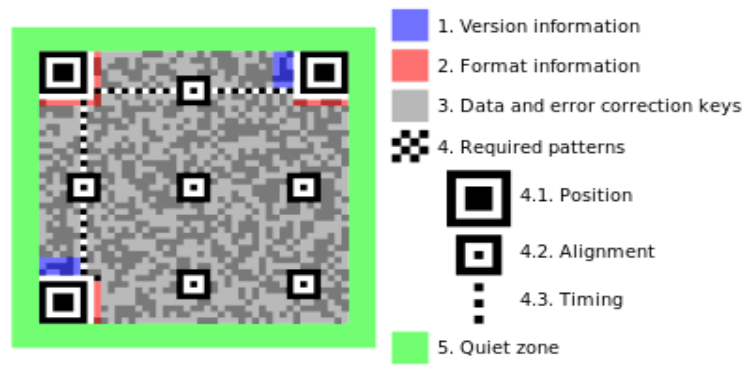

Fig1: QR code structure
Methods [6] that modify the luminance or color of image pixels and methods that replace QR modules along with this there have been several efforts to improve the appearance of such embedding of color images in QR code [7]. The method presented in [8] represents luminance intensity of the QR code, on finding the best group of QR modules to substitute by the image or logo in the QR code. To introduce the image without affecting the decoding robustness, in the padding regions other methods take advantage of unused modules. If images are superimposed over finder or alignment patterns, then the probability of correct decoding will be decreased. Once, logos and images are located at the center of the QR code then it imposes restrictions in the location of modified modules; in general these approaches do not take advantage of the codeword generation process.

This problem was addressed by newly developed techniques without reducing the correction capacity which manipulates the Reed Solomon encoding procedure of QR code. Then the reliability of embedding is being regulated which is based on the average luminance of image pixels and two user defined thresholds by the modified pixels in the code. With respect QR blocks are still visible but the visual impact is reduced.

There will be increase in the probability of detection error because of embedding of image pixels introduce changes in the luminance of the code .Due to this change it introduce disturbance in the binarization thresholds. This paper aims at the above presented goals by showing QR images with minimum distortion. Here the algorithm proposed is based on the selection of a set of modified pixels using a multiresolution halftoning mask. Corresponding pixel luminance and concentration of pixels are optimized to minimize a visual distortion.

\section{EXISTING WORK}

QR codes have already overtaken the classical barcode in popularity in various areas. Because of the diversity and extendibility of QR codes the barcodes are repulsive than $\mathrm{QR}$ codes. Good characteristic of QR code is that they do not need to be scanned from one particular angle and regardless of their positioning QR codes can be read. Scanners of QR codes are capable of determining the correct way to decode the image due to the three specific squares that are positioned in the alignment block and the corners of the symbol of QR code. At the start QR codes were used by vehicle manufacturers for tracking parts. After a while, companies began to see the variety of different use cases for QR Codes. The most popular commercial use for QR Codes is in the mobile communication industry.

\section{RELATED WORK}

Automatic identification and data capture (AIDC) is the use of technology which allows automatic feeding of information in the computer, or other VLSI or micro-processor controlled system, instead of reloading of information to manual methods. AIDC system can operated independently without taking help from human beings to do the operations like 
collection of information from various fields, holding the same for further requirement .Generally in the many industries their crucial operation and analysis are being done in AIDC mode [1].

Soma. V, Vijaya Lakshi. P et al. [3] in their work presents a High Capacity Color Barcode framework used for capturing color images is proposed by exploiting the spectral diversity afforded respectively by the Cyan; Magenta and yellow print colorant channels and the Red, Green and Blue complimentary channels. Once the color images are captured then, by transforming the independent data in the Cyan, Magenta, and Yellow print colorant channels and data from the complimentary R, G, and B channels is decoded. It is achieved a three-fold increase in the data rate. The effect of cross-channel interference among the print colorant and capture color channel to reduce the effect of cross channel. The probability of correct decoding will be severely decreased. The probability of correct decoding will be severely decreased Algorithm is used that estimates the parameters from regions encoding the data itself [2] [3].

S. Ono, K. Morinaga, and S. Nakayama et al [9] proposed a method to improve on our previously proposed but problem pursue innovation for generating animated and illustrated Quick Response (QR) codes, here they proposes a method which formulates the animated QR code generation problem. Practical experiments demonstrate that the proposed method can generate animated $\mathrm{QR}$ codes involve a maximum of eight illustrations moving inside the code which maintaining decoding feasibility and smooth illustration movement.

During a studied time interval bit error rate or BER is the basic idea of comparison between two pictures to perform the number of bit errors divided by the total number of transferred bits. It is possible to compare two images in order that measure quality of them in image processing. The proposed method [10] shows the use of adaptation of brightness in image to get lower BER. Here they focus on QR Code image, compare with QR Code is overlapped by a portrait and test by decode them.

This paper introduces the concept of QR codes, an automatic method to insert vital information using QR codes into color images. The embedding methods are designed to be compatible with standard decoding applications and can be applied to any color or gray scale image with full area coverage .There are two components. First is the use of halftoning techniques for the selection of modified pixels to break and reduce the coarse square structure of the QR code and second is the luminance level to which the pixels are to be transformed in such a way that it should not visible to naked eye on the color image. Further to decode the QR code from the color image with minimum distortion errors [5].

The Qart code which is designed with distortion rate is 0.4152 , the distortion rate for the existing system is 0.3989 while here proposed system will have distortion rate less than existing near about 0.3889. Graphical representation of distortion metrics is shown in figure.

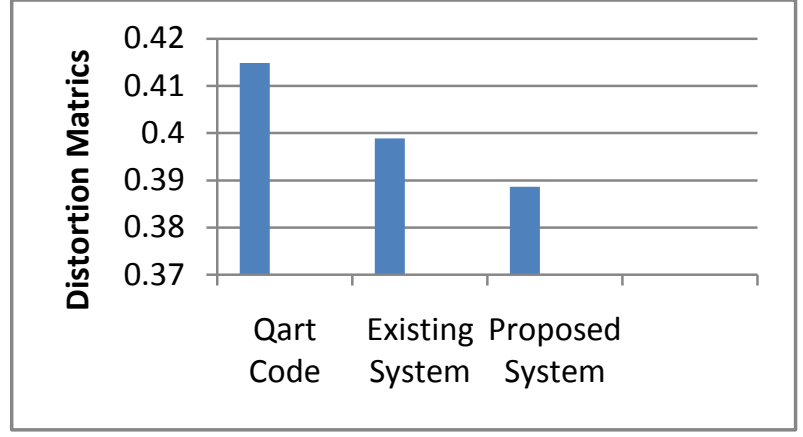

\section{Fig2: Distortion metrics}

H.K.Chu, C.S.Chang, R-R.Lee and N.J Mitra et al [11] in their work shows a QR code is a popular form of barcode pattern that is ubiquitously used for linking advertisements and also for product information to tag barcode. For an application barcode is required to keep the patterns machine readable. Probability distributions of replacement of modules with other modules are learning in pattern readability function. After that, given a text tag, they express the input image in terms of the learned dictionary where source text will be encoded.

Many approaches have been proposed for image embedding in $\mathrm{QR}$ codes over the last few years

\subsection{Procedure of $Q R$ code embedding}

In the embedding the information bits of the input $\mathrm{QR}$ code image is encoded into luminance value of the image while performing this encoding, value of luminance changes. For light region average luminance is increases and for dark region it is decreases. Central portion of QR code is used to hide the information. Multiresolution halftoning and color optimization are the two techniques used for image embedding.

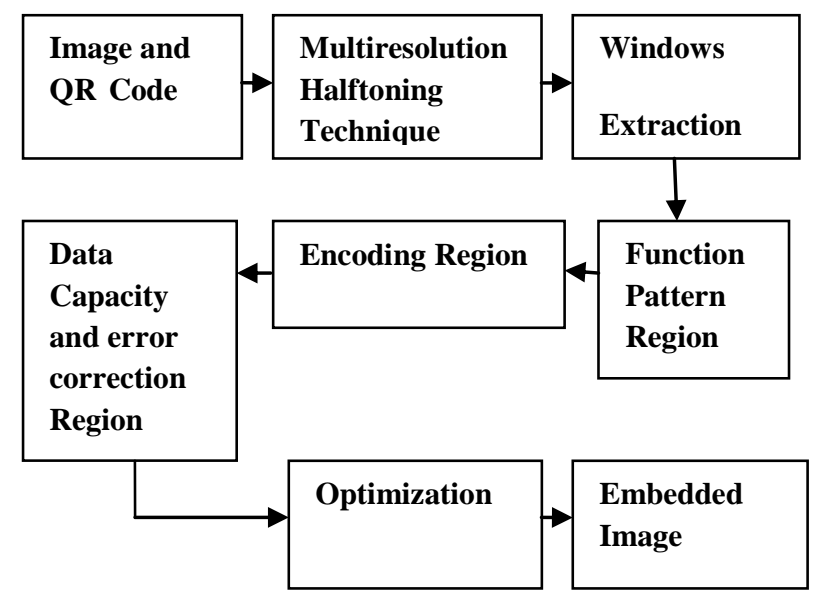

Fig 3: QR code embedding

This system takes QR code and image as an input. By using halftoning masking binarization is performed on the input image with accuracy. After the binarization, the window area is divided as well as extracted. In QR code embedding when window extraction is completed then it selects function pattern region of $\mathrm{QR}$ code. After selection of function pattern region, the image is embedded in the encoding. There will be increase in the probability of detection error because of embedding of image pixels Second challenge is the problem of using the entire area of the code where they inserts image or logo. Then by simply replacing information modules with the desired image this cannot achieved since, the number of 
modules that can be replaced is at most proportional to the correction capacity of the code.

\subsubsection{Input image and $Q R$ code}

To embed QR code into color image inputs are original image QR code.

\subsubsection{Multiresolution halftoning technique}

Proposed technique by M. Mukherjee and G.Sharma et al [12] gives a Progressive halftoning In-Place (P-HIP) method. It combines multilevel error diffusion and screening to progressively halftone and render a grayscale image with gradually increasing resolution. For image storage it uses only the binary display memory and thereby eliminates the need for additional memory to store contone image data; hence it offers improved memory efficiency.

\subsubsection{Window extraction}

Images are divided into local window and get extracted which is required for image optimization.

\subsubsection{Function pattern region}

In the region the most essential module and route to locate, rotate and align the QR code are the finder and alignment structures. For finding the sampling grids from which code words are extracted alignment patterns are used and they are easily identifiable as concentric square structures evenly distributed along the code area.

\subsubsection{Encoding region:}

It is the code area delimited by finder pattern, in the encoding region parity modules, encoding information and decoding information is stored. Encoding region is divided into code words where a block of $8 \mathrm{QR}$ modules contains in the code words.

\subsubsection{Data capacity and error correction region:}

QR code version and error correction level are used to identify their different version of QR code. It determines its size and which starts from $21 \times 21$ modules for version 1 up to $177 \times 177$ for version 40 of $\mathrm{QR}$ code. In $\mathrm{QR}$ code for error correction Reed Solomon code used by QR code and these are L, M, Q and $\mathrm{H}$ error correction level which permits error correcting as $7 \%, 15 \%, 20 \%$ and $30 \%$ of code words in error respectively.

\subsection{QR code decoding}

The input to reader application is embedded QR image. Decoding is then further process by calculating threshold, After calculating the threshold the mean block binarization method is used to convert image into binary format then the required sample is selected by calculating its luminance. Lastly the codeword is extracted through sampling grid information. The code is corrected \& retrieves during decoding [2]

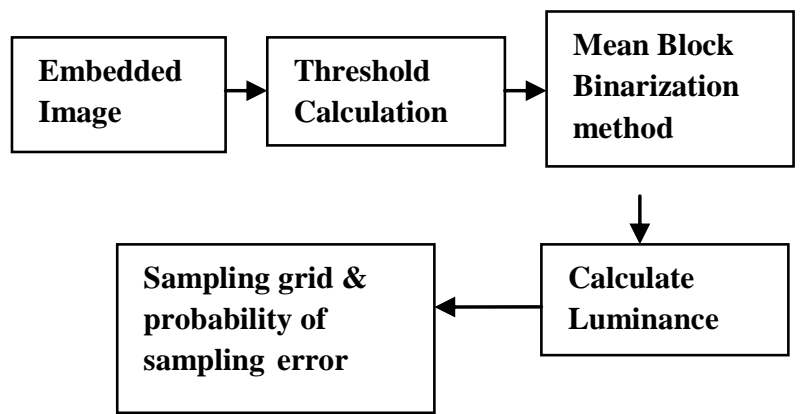

\subsubsection{Calculation of threshold}

The gray scale image gained by thresholding which in turns gives binary image Ip. Then this binary images are required at the time of binarization and the important thing is that decoding process is begins with binarization. Thresholds can be calculated globally using all the pixels in the image or locally consider only reduced windows. Binary image $I_{P}$ is obtained by thresholding the image $Z_{i, j}$

$$
\begin{aligned}
\text { Ip } & =1 \text { if } Z i, j>\theta i, j \\
& =0 \text { if } Z i, j \leq \theta i, j
\end{aligned}
$$

Where, $\mathrm{Zi}, \mathrm{j}$ is the gray scale image captured by camera, $\Theta i, \mathrm{j}$ is threshold which is assigned to $[\mathrm{i}, \mathrm{j}]$ pixel in the image and Ip is the output binary image.

\subsubsection{Binarization method based on mean block}

During binarization, the black and white pixels are obtained by segmenting the gray scale QR code image. There is one open source Zxing library is the one of the most popular libraries for generation and reading of QR code. Binary image Ip determines the center of the QR modules. Gonzalo J. Garateguy, Gonzalo R. Arce, Daniel L. Lau and Ofelia P. Villarreal et al [2] through a hybrid method the thresholds used here, are calculated. Where it uses the average luminance in a set of overlapping square windows. The $\mathrm{D} u, v$ of $8 \times 8$ non-overlapping blocks pixels is obtained by dividing captured image, then the average luminance in overlapping sub windows of $5 \times 5$ blocks is evaluated by using

$$
\begin{aligned}
& \mathrm{m}=\mathrm{u}+2 \quad \mathrm{n}=\mathrm{v}+2 \\
& \mathrm{Tu}, \mathrm{v}=\frac{1}{25 \times 64} \sum \sum \sum z[k, l] . \\
& \mathrm{m}=\mathrm{u}-2 \quad \mathrm{n}=\mathrm{v}-2 \quad(\mathrm{k}, 1) \in \mathrm{Dm}, \mathrm{n}
\end{aligned}
$$

The averages calculated for each block $\mathrm{D} u, v$ are assigned to the image pixels in the block as $\theta i, j=T u, v$ for $[i, j] \in \mathrm{D} u, v$. For developing the probability of error model and the QR embedding algorithm it is important.

\subsubsection{Sampling Grid}

When the binary image $I_{P}$ is obtained, by sampling on a grid estimated using finder and alignment patterns codeword are extracted. [13] After the extraction of codeword the next step is drawing parallel lines between the estimated centers of finder and alignment patterns and the spacing between lines, the points in this grid are generated which estimate the QR module width. For larger code sizes, as the center of QR code it contains information; to compensate local geometric distortions there a multiple sampling grids are used [14]. For determining binary value the luminance around the center of the module should be perfectly defined.

\subsubsection{Probability of sampling error}

QR code is extracted from color image once, the process of binarization and sampling grid is completed. To check the similarity between the extracted image and original image here preference will be given to comparisons between extracted image and original image. Probability of sampling error defines as, at any pixel in the $\mathrm{QR}$ modules the probability of sampling the incorrect binary values. These errors are affected by the various other factors. In the QR code their distribution of pixels and luminance in the image and luminance transformation parameter which affects the probability of sampling errors most.

Fig4: QR code decoding 


\section{CONCLUSION}

We reviewed the most of recent development in $\mathrm{QR}$ code as we gone through literature. A novel contribution of this paper is the use of multi resolution halftone masks to distribute the modified pixels .With this multiresolution the memory efficiency increases as it stores contone image and outputs image by using of single buffer frame. Multiresolution allows controlling the tradeoff between image qualities and decoding robustness. With this mutiresolution algorithm the resulting half toned images are comparatively better in quality than other algorithm. In this paper it proposes to embed color image into $\mathrm{QR}$ code with minimum visual distortion than existing.

In next generation it will be possible to embed any gray scale or colored video into the QR codes.

The main target lies in the accurate analysis and practical application of one or more of the outlined attacks on a given target QR code. Furthermore, it should be investigated which parts of a $\mathrm{QR}$ code are the easiest to attack.

\section{ACKNOWLEDGMENTS}

I take this opportunity to express my heart-felt gratitude to my guide, Prof. S. B. Borse, for his constant encouragement, wonderful technical guidance and support throughout the course. I sincerely thank Prof. S. B. Bagal, HOD of Electronics \& Telecommunication Department for his advice and support during course of this work.

\section{REFERENCES}

[1] Information Technology-Automatic Identification and Data Capture Techniques-Bar Code Symbology QR Code, Int. Org. Standardization, Geneva, Switzerland, ISO/IEC 18004, 2006.

[2] Gonzalo J. Garateguy, Gonzalo R. Arce, Daniel L. Lau and Ofelia P. Villarreal "QR Images: Optimized Image Embedding in QR Codes," Member IEEE in July-2014

[3] Soma. V, Vijaya Lakshmi. P, Anna University Chennai, Tamilnadu, India "Per-Colorant-Channel High Capacity Color Barcodes: An Interference Cancellation Framework" ISSN, Vol.2, Special Issue 1, March 2014

[4] Max E. Vizcarra Melgar,Alexandre Zaghetto,Bruno Macchiavello Anderson C,A.Nascimento, "Colored Quick Response Codes", $2^{\text {nd }}$ International Conference on Consumer Elctronics,vol 3,pp.321-325,September 2012
[5] Y. Lin, Y. Chang, and J. Wu, "Appearance-based QR code beautifier," IEEE Trans. Multimedia, vol. 15, no. 8, pp. 2198-2207, Dec. 2013.

[6] M. Hara, M. Watabe, T. Nojiri, T. Nagaya, and Y. Uchiyama, "Optically readable two-dimensional code and method and apparatus using the same," U.S. Patent 5 726 435, Mar. 10, 1998.

[7] Mu Zhang ,Dan Yao ,Qian Zhou , "The Application and Design of QR code in Scenic Spot's eTicketingSystem", International Journal of Science and Technology, Vol 2, Pp817-821, December 2012

[8] Munoz-Mejja's, Ivan Gonzalez-Diaz and Fernando Diazde-Maria, "A Low-complexity Pre-processing System for Resorting Low Quality QR Code Images", IEEE Transction on consumer Electronics, Vol57,PP 13201328,August 2011.

[9] S. Ono, K. Morinaga, and S. Nakayama, "Twodimensional barcode decoration based on real-coded genetic algorithm," in Proc. IEEE CEC, Jun. 2008, pp. 1068-1073.

[10] D. Samretwit and T. Wakahara, "Measurement of reading characteristics of multiplexed image in QR code," in Proc. 3rd Int. Conf. Intell. Netw. And Collaborative Syst., 2011, pp. 552-557.

[11] H.-K. Chu, C.-S. Chang, R.-R. Lee, and N. J. Mitra, "Halftone QRcodes," ACM Trans. Graph., vol. 32, no. 6, p. 217, 2013.

[12] Mithun Mukherjee and Gaurav Sharma "A Multiresolution Halftoning Algorithm for Progressive Display", Rochester Institute of Technology, Rochester, USA Color Imaging X: Processing, Hardcopy and Applications, edited by Reiner Eschbach, Gabriel G. Marcu Proc. of SPIE-IS\&T Electronic Imaging,SPIE Vol. 5667 (C) 2005

[13] RouA-Lin,Feng Yuan and Geng Ying, "QR code image detection using run-length coding", $18^{\text {th }}$ international Conference on Computer science and Network Technology.vol4,pp 2130-2134,November 2011

[14] Y.Shantikumar Singh, B.Pushpa Devi and Kh.Manglem Singh, "Different Techniques on Digital Image Watermarking Scheme", International Journal of Engineering Research, vol 2, pp.193-199, July 2013 